\section{Diffuse interstitial lung disease due to AA amyloidosis}

\author{
Carole Planes, Dieter Kleinknecht, \\ Michel Brauner, Jean-Paul Battesti, \\ Jean-Louis Kemeny, Dominique Valeyre
}

\begin{abstract}
A man developed interstitial lung disease and nephrotic syndrome due to $A A$ amyloidosis. There was no evidence of an underlying disease predisposing to amyloidosis.
\end{abstract}

Amyloidosis has been classified according to the biochemical properties of amyloid. ${ }^{1}$ The $\mathrm{AA}$ and $\mathrm{AL}$ forms of amyloidosis are both common, but respiratory manifestations are usually observed in $\mathrm{AL}$ amyloidosis. We report a case of diffuse interstitial lung disease due to AA amyloidosis.

\section{Service de \\ Pneumologie C Planes \\ J-Paul Battesti \\ D Valeyre}

Service de Radiologie

93105, Montreuil,
M Brauner

Service de

Anatomopathologie

J-L Kemeny

Hôpital Avicenne,

125 route de

Stalingrad,

93009 Bobigny, France

Service de

Néphrologie,

Centre Hospitalier,

56 boulevard de la

Boissière, France

D Kleinknecht

Reprint requests to: Dr Valeyre

Accepted 22 August 1991

\section{Case report}

A 71 year old Jewish man of North African origin was admitted to hospital because of recent exertional dyspnoea and a weight loss of $4.5 \mathrm{~kg}$. He had been a moderate smoker until the age of 55, but had no prior health problems. Physical examination showed no cardiac or pulmonary abnormality, but his spleen and liver were enlarged. Blood tests showed: haemoglobin $10 \mathrm{~g} / \mathrm{dl}$, normal white blood cell and platelet counts, erythrocyte sedimentation rate 115 in one hour, and hypergammaglobulinaemia (gammaglobulin $12 \mathrm{~g} / \mathrm{l}$ ), normal coagulation, and normal values in liver function tests. He had a nephrotic syndrome with a serum concentration of albumin of $20 \mathrm{~g} / \mathrm{l}$,

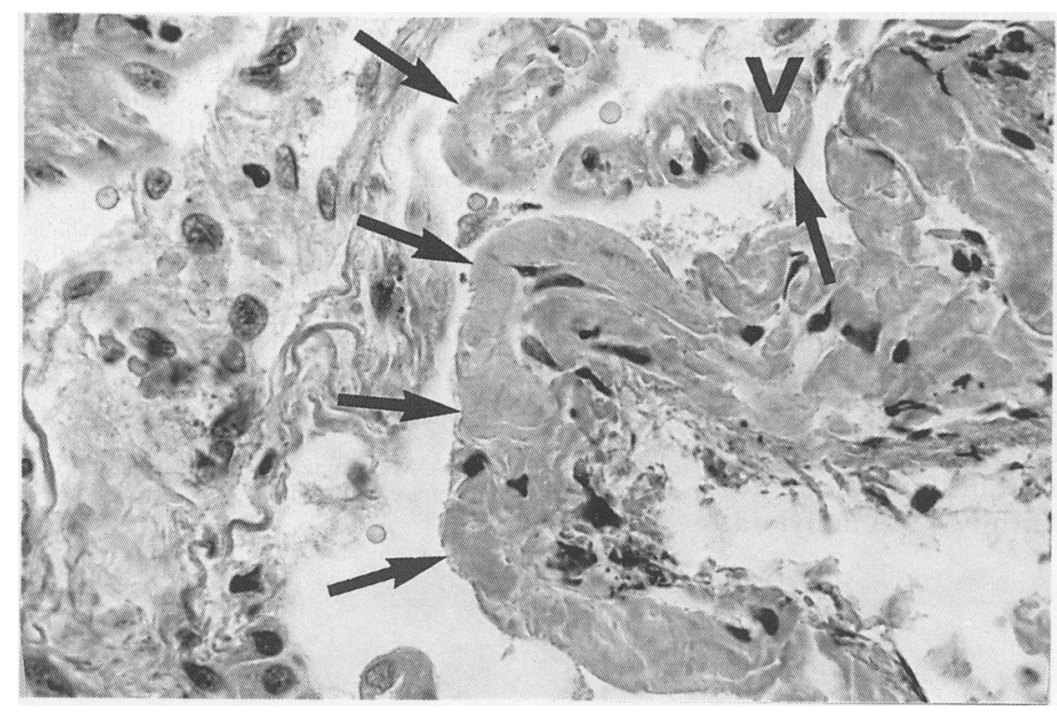

Figure 1 Transbronchial biopsy specimen, showing evidence of diffuse distribution of amyloid deposits (arrows) in alveolar walls and vessels $(V)$.

(Haematoxylin-phloxin-safran.) proteinuria (protein loss of 5-10 g/day) without haematuria, and severe renal failure (creatinine clearance $25 \mathrm{ml} / \mathrm{min}$ ). Serum and urine electrophoresis and immunoelectrophoresis did not show an increase in monoclonal immunoglobulin. Arterial blood gas analysis showed hypoxaemia $(8.6 \mathrm{kPa})$ and normocapnia. A chest radiograph showed diffuse reticular shadowing in both lung fields, especially in the lower left lobe, and a few bullae. The pleura, mediastinum, and cardiothoracic ratio were normal. Lung volumes were increased (total lung capacity $127 \%$ of predicted value, vital capacity (VC) $120 \%$, residual volume $161 \%$ ), and he had severe airways obstruction $\left(\mathrm{FEV}_{1} /\right.$ VC $43 \%$ ). Transfer factor corrected for lung volume was reduced $(0.54 \mathrm{mmol} / \mathrm{min} / \mathrm{kPa} / 1$, $33 \%$ of predicted). High resolution computed tomography of the thorax showed a diffuse interstitial pattern, with small nodules in posteroinferior areas and linear shadows forming a network resembling thickening of interlobular septa, and small areas of centrolobular emphysema. Renal ultrasonography showed irregular kidneys, but of normal size, without any urological obstruction. The electrocardiogram and echocardiogram were normal.

A percutaneous renal biopsy showed massive glomerular and arteriolar deposits of amyloid; this was confirmed by Congo red staining, which showed green birefringence under polarised light. Pretreatment with potassium permanganate abolished the uptake of Congo red. Fibreoptic bronchoscopy showed macroscopically normal appearances, but distal bronchial and transbronchial biopsy specimens from the left lower lobe showed amyloid deposits in interalveolar septa, septal vessel walls, and distal bronchioles (fig 1). No tumour or lymphoplasmocytic infiltrates were seen near the deposits, which were potassium permanganate sensitive. Immunohistochemical staining was performed on both renal and lung biopsy specimens to detect the presence of AA protein or immunoglobulin light chains. Rabbit antibodies against human AA protein (Calbiochem, La Jolla, California) or human lambda or kappa chains (Dakopatts, Glostrup, Denmark) were used, followed by the peroxidase-antiperoxidase (PAP) technique (PAP kit system, Dakopatts, Glostrup, Denmark). ${ }^{2}$ Staining was strongly positive for AA protein in both renal and transbronchial tissues but negative for immunoglobulin light chains, the main component of AL amyloidosis (fig 2).

The search for a predisposing cause for amyloidosis produced no evidence of any chronic infectious process (tuberculosis, syphilis, bronchiectasis); inflammatory disease, such as rheumatoid arthritis, systemic lupus erythematosus, Behçet's syndrome, or a familial or personal history of periodic fever; and no evidence of Hodgkin's disease or other neoplastic disease (normal results of gastric fibroscopy and colonoscopy, pelvic and renal ultrasonography and urinary cytology).

A 24 month follow up with no treatment showed no progression of dyspnoea or radiological abnormalities, but renal function worsened and the nephrotic syndrome persis- 
Figure 2 Transbronchial biopsy specimen, showing (A) immunohistochemical staining positive for $A A$ protein in alveolar walls (right part of the photomicrograph); and (B) staining negative for kappa immunoglobulin light chains and the presence of anthracoid deposits.

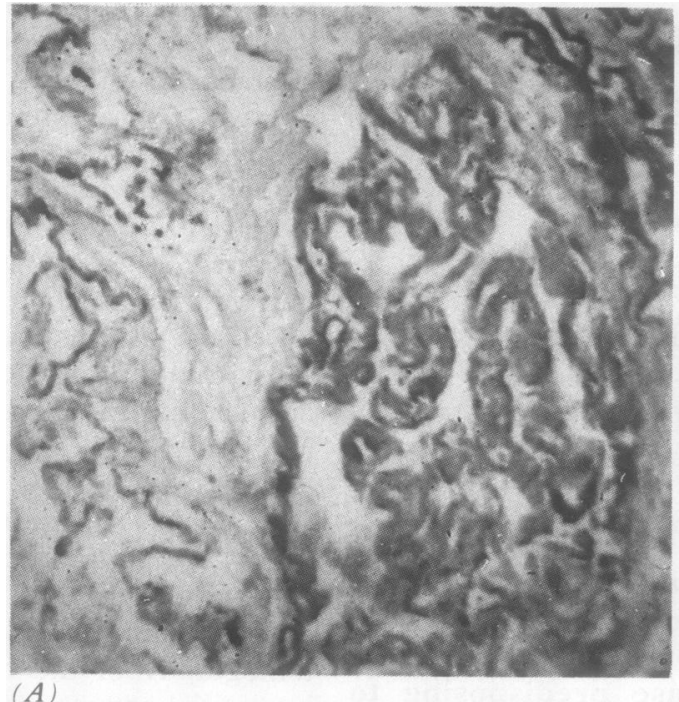

deposits were evident inside interalveolar septa, in septal vessel walls, and in bronchiole walls.

Amyloidosis of the lower respiratory tract exists as tracheobronchial amyloidosis, including pseudotumoral masses and multifocal submucosal plaques, and parenchymal amyloidosis, in a nodular (single or multiple) or diffuse interstitial form. Tracheobronchial amyloid and pulmonary nodules are generally localised to the respiratory tract, whereas the diffuse interstitial type is usually due to systemic amyloidosis. Lung infiltration by AA protein is rarely evident on a chest radiograph. ${ }^{56}$ Only two similar cases have been reported, one associated with Hodgkin's disease, ${ }^{7}$ and the other with systemic lupus erythematosus. ${ }^{8}$ Small and localised AA amyloid deposits in pulmonary arterioles, however, is less exceptional but usually remains clinically and radiologically asymptomatic. ${ }^{9}$ The absence of clinical or radiological progression during 24 months is unusual as diffuse lung disease is usually progressive and rapidly fatal in both AL and AA amyloidosis, though eight years' survival with diffuse interstitial pulmonary primary amyloidosis has been reported. ${ }^{10} \mathrm{AA}$ systemic amyloidosis is usually due to an underlying disease, either infectious, inflammatory, or neoplastic; but this could not be shown in our case even with two years' observation, though quiescent or occult disorders, such as Castelman's disease, could not be completely excluded.

We are grateful to Mme D Droz (Service d'anatomopathologie, Hôpital Necker, Paris), who performed the immunohistochemical staining, and to Mrs Blanchard for kindly typing the text.

1 Glenner GG. Amyloid deposits and amyloidosis. $N$ Engl J Med 1980;302:1283-92, 1333-44.

ted. C reactive protein was increased at $6 \times 10^{-3}$ $\mathrm{mmol} / \mathrm{l}$ (normal value $<2.6 \times 10^{-4} \mathrm{mmol} / \mathrm{l}$ ) No clinical evidence of a predisposing disease emerged.

\section{Discussion}

The diagnosis of systemic AA amyloidosis was based in this case on histopathological and immunohistochemical findings. The singularity of this case results from the rarity of radiological pulmonary infiltration due to AA amyloidosis, the slowly progressive course of the lung disease, and the lack of evidence both initially and during follow up of any underlying predisposing disease. Pulmonary interstitial infiltration by AA amyloidosis was suggested by the potassium permanganate technique, which inhibited Congo red staining. As reduction of Congo red staining may also be seen with immunoglobulin derived amyloidosis, ${ }^{34}$ we used immunohistochemical methods to establish the diagnosis firmly. ${ }^{2}$ AA amyloid
2 Noel LH, Droz D, Ganeval D. Immunohistochemical characterization of renal amyloidosis. Am J Clin Pathol 1987;87:756-61.

3 Wright JR, Calkins E, Humphrey RL. Potassium permanganate reaction in amyloidosis. A histologic method to 1977;36:274-81.

4 Hui AN, Koss MN, Hochholzer L, Wehunt WD Amyloidosis presenting in the lower respiratory tract. Clinico-pathologic, radiologic, immunohistochemical and histochemical studies on 48 cases. Arch Pathol Lab Med 1986;110:212-8.

5 Cordier JF, Loire R, Brune J. Amyloidosis of the lower respiratory tract. Clinical and pathologic features in a series of 21 patients. Chest 1986;90:827-31.

6 Smith RRI, Hutchins GM, Moore GW, Humphrey RI. Type and distribution of pulmonary parenchymal and vascular amyloid. Am J Med 1979;66:96-104.

7 Pfizenmeyer P, Massins S, Fargeot P, Bonneau R, Michiels $R$, Jeannin $L$. Amyloidose pulmonaire de type AA associée

8 Nomura S, Kumagai N, Kanoh T, Uchino H, Kirihara J. Pulmonary amyloidosis associated with systemic lupus erythematosus. Arthr Rheum 1986;29:680-2.

9 Celli BR, Rubinow A, Cohen AS, Brochy JS. Patterns of pulmonary involvement in systemic amyloidosis. Chest 1978;74:543-7.

10 Kanada DJ, Sharma OM P. Long term survival with diffuse interstitial pulmonary amyloidosis. Am J Med 1979; 67:879-82. assist in differentiating forms of this disease. Lab Inves à une maladie de Hodgkin. Rev Med Interne 1988;9:524-7. 